\title{
TR34/L98H Mutation in CYP51A Gene in Aspergillus fumigatus Clinical Isolates During Posaconazole Prophylaxis: First Case in Korea
}

\author{
Hyeon-Jeong Lee $\mathbb{D} \cdot$ Sung-Yeon Cho $\mathbb{D} \cdot$ Dong-Gun Lee $\cdot$ Chulmin Park • \\ Hye-Sun Chun • Yeon-Joon Park
}

Received: 25 January 2018/Accepted: 12 May 2018/Published online: 1 June 2018

(C) The Author(s) 2018

\begin{abstract}
Azole resistance in Aspergillus fumigatus is an emerging problem, especially in immunocompromised patients. It has been reported worldwide, including in Asia, but has not yet been reported in Korea. Here, we report a case of invasive pulmonary aspergillosis (IPA) caused by azole-resistant A. fumigatus that developed in a hematopoietic stem cell transplantation recipient during posaconazole prophylaxis for immunosuppressive therapy of graft-versushost diseases. We identified TR34/L98H/S297T/
\end{abstract}

Handling Editor: Sudha Chaturvedi.

H.-J. Lee · S.-Y. Cho $(\varangle) \cdot$ D.-G. Lee

Division of Infectious Diseases, Department of Internal Medicine, The Catholic Blood and Marrow

Transplantation Center, Vaccine Bio Research Institute, Seoul St. Mary's Hospital, College of Medicine, The Catholic University of Korea, 222, Banpo-daero, Seochogu, Seoul 06591, Republic of Korea

e-mail: cho.sy@catholic.ac.kr

H.-J. Lee

e-mail: lhj2113411@hanmail.net

D.-G. Lee

e-mail: symonlee@catholic.ac.kr

H.-J. Lee · S.-Y. Cho - D.-G. Lee · C. Park · H.-S. Chun Vaccine Bio Research Institute, Seoul St. Mary's Hospital, The Catholic University of Korea, 222, Banpodaero, Seocho-gu, Seoul, Republic of Korea

e-mail: micropak@catholic.ac.kr

H.-S. Chun

e-mail: onlyjhs75@naver.com
F495L mutation in the CYP51A gene of A. fumigatus clinical isolate obtained from bronchial washing fluid. Minimal inhibitory concentrations for itraconazole, voriconazole, and posaconazole were $>16,1$, and $4 \mu \mathrm{g} / \mathrm{mL}$, respectively. While IPA improved partially under voriconazole treatment, the patient died from carbapenemase-producing Klebsiella pneumoniae bacteremia. Further epidemiological surveillance studies are warranted.

Keywords Aspergillus fumigatus · Azoles · Posaconazole $\cdot$ CYP51A

S.-Y. Cho - D.-G. Lee

The Catholic Blood and Marrow Transplantation Center, Seoul St. Mary's Hospital, College of Medicine, The Catholic University of Korea, 222, Banpo-daero, Seochogu, Seoul 06591, Republic of Korea

Y.-J. Park

Department of Laboratory Medicine, Seoul St. Mary's Hospital, College of Medicine, The Catholic University of Korea, 222, Banpo-daero, Seocho-gu, Seoul 06591, Republic of Korea e-mail: yjpk@catholic.ac.kr 


\section{Introduction}

Aspergillus infections including invasive aspergillosis (IA) mainly affect immunocompromised patients, such as hematopoietic stem cell transplantation (HSCT) recipients or patients undergoing immunosuppressive therapy. Due to the increased number of immunocompromised patients, the incidence of IA has increased over the past three decades. Among Aspergillus species (spp.), Aspergillus fumigatus remains the most common species in all pulmonary syndromes. Although diagnostic advances and new triazole antifungal drugs have now been established, mortality rates associated with IA remain high and range between 28.5 and $50 \%$ [1].

As triazole drugs have been the mainstay of both treatment and prevention of IA since the 1990s, there were concerns about the increasing possibility of that azole-resistant Aspergillus spp. or azole breakthrough IA by expanding the usage of triazoles [2]. Since the first azole-resistant A. fumigatus was found both in the Netherlands and in Italy in 1998 [3], azole resistance in A. fumigatus isolates has been found in almost every European country, the Middle East, Asia, Africa, Australia, and most recently, North and South America. Substitution of leucine 98 with histidine in the CYP51A gene in combination with a 34-bp tandem sequence in the promoter gene (TR34/L98H) is a dominant resistance mechanism thought to be acquired from the environment [4-6]. Reported rates of azole-resistant $A$. fumigatus vary from 0.6 to $27.8 \%$ $[5,7]$. However, there has been no evidence that azoleresistant $A$. fumigatus is a problem in Korea.

\section{Case Report}

A 27-year-old man who received second matched unrelated donor HSCT for relapsed acute lymphoblastic leukemia (ALL) $(\mathrm{D}+210)$ was hospitalized for treatment of aggravated grade IV skin graft-versushost disease (GVHD). The patient started high-dose steroid therapy ( $>1 \mathrm{mg} / \mathrm{kg}$ per day of prednisolone) for GVHD and continued taking posaconazole (PCZ) tablets $(300 \mathrm{mg} \mathrm{q} 12 \mathrm{~h}$ for 2 doses and then $300 \mathrm{mg}$ once daily) which had been administered for 77 days from the outpatient clinic for fungal prophylaxis in severe GVHD. During the high-dose steroid treatment, more than $10 \%$ of blasts were detected in peripheral blood cell counts, and ALL again relapsed after the second HSCT was confirmed.

On the 7th day of hospitalization (HD 7), the patient suddenly complained of fever (maximum body temperature $38.6{ }^{\circ} \mathrm{C}$ ) and dyspnea, and then, his blood pressure dropped to $84 / 43 \mathrm{mmHg}$. Oxygen demand was gradually increased, and tracheal intubation was performed. At that time, it was the seasonal influenza epidemic, and rapid influenza antigen test resulted in influenza A positive and chest X-ray showed infiltrations in the right lung fields (Fig. 1). Therefore, the first impression was influenza A pneumonia with septic shock. Considering the possibility of combined other nosocomial bacterial pneumonia or atypical pneumonia, not only peramivir (600 mg once), but also cefepime ( $2 \mathrm{~g} \mathrm{q12} \mathrm{h}$ ), levofloxacin (750 mg once daily), and teicoplanin (400 mg q12 h for 3 doses and then $400 \mathrm{mg}$ once daily) were administered. On the chest, low-dose computed tomography (LDCT) performed, and multifocal ground-glass opacities (GGOs) accompanied by peribronchial consolidations and illdefined centrilobular nodules in both lungs were observed (Fig. 2). On the 3rd day of fever onset (HD 10), bronchoscopy was performed. His condition recovered rapidly and intubation was removed on the 4th day. Blood and sputum cultures, Streptococcal pneumoniae and Legionella urinary antigen tests, Mycoplasma serum IgM/IgG tests, and serum galactomannan assay were all negative.

On the 4th day after bronchoscopy (HD 11), Aspergillus spp. was cultured from the bronchial washing fluid specimen. It was thought to be a true

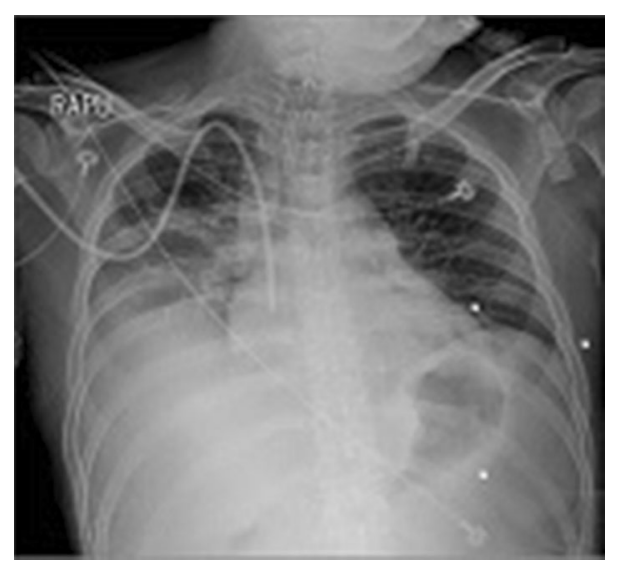

Fig. 1 Chest X-ray showed infiltrations in right upper and lower lung fields 

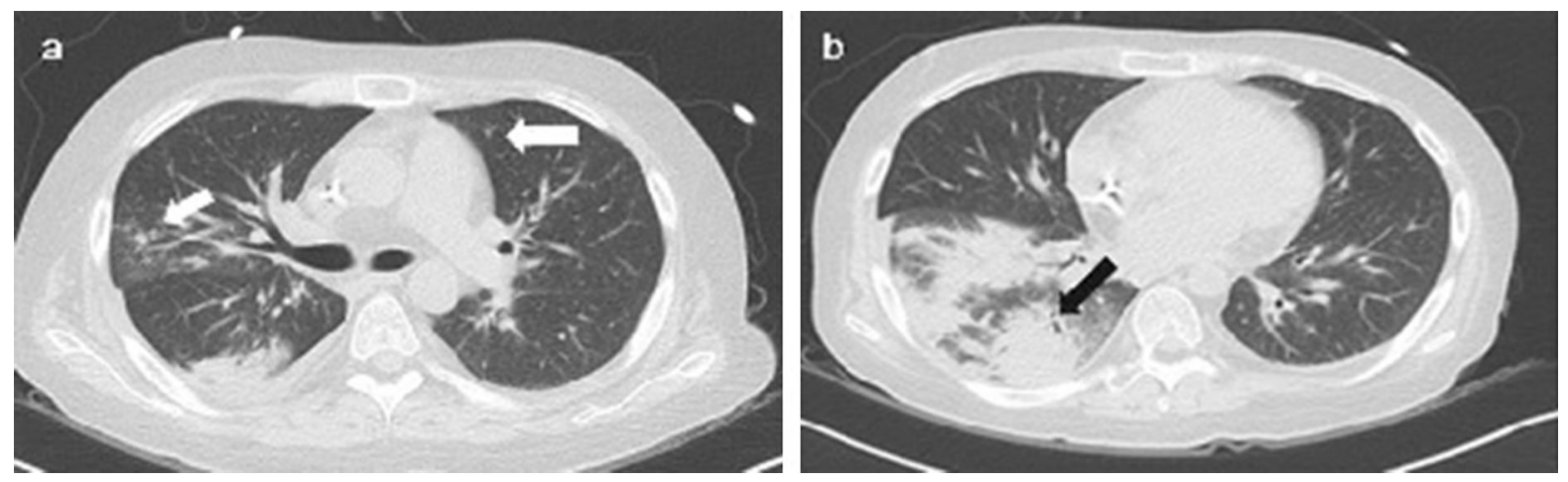

Fig. 2 Low-dose chest computed tomography. a Ill-defined centrilobular nodules in both lungs (white arrows). b Peribronchial consolidation that accompanied by ground-glass opacities

pathogen because chest LDCT revealed consolidations with surrounding GGO and nodules consistent with fungal pneumonia that developed during the course of long-term maintenance of high-dose steroid treatment. According to revised European Organization for Research and Treatment of Cancer/Mycosis Study Group (EORTC/MSG) criteria [8], the patient was diagnosed as a culture-positive invasive pulmonary aspergillosis (IPA) with probable category. At the time of diagnosis of IPA, the patient had been receiving $\mathrm{PCZ}$ for 87 days, maintaining a therapeutic range of PCZ serum concentrations (1048-2232 ng/ $\mathrm{mL}$ ). Therefore, this case was considered as PCZ breakthrough IA.

PCZ was changed to intravenous voriconazole (VCZ) (loading dose $6 \mathrm{mg} / \mathrm{kg} \mathrm{q} 12 \mathrm{~h}$, then $4 \mathrm{mg} / \mathrm{kg}$ $\mathrm{q} 12 \mathrm{~h}$ ). Other antibiotics were also discontinued because there was no evidence of other bacterial pathogens. Eight days later, Aspergillus spp. was finally confirmed as A. fumigatus by internal transcribed spacer (ITS) sequencing and PCR of the $\beta$ tubulin gene as follows. Their entire ITS regions were amplified using the primers of ITS1-F_KYO2 $\left(5^{\prime}\right.$ TAGAGGAAGTAAAAGTCGTAA- $\left.3^{\prime}\right)$ and ITS4 (5'TCCTCCGCTTATTGATATGC- $3^{\prime}$ ), as previously described [9]. $\beta$-tubulin PCR was performed by bt $2 \mathrm{a}$ $\left(5^{\prime}\right.$-GGTAACCAAATCGGTGCTGCTTTC- $\left.3^{\prime}\right)$ and bt2b (5'-ACCCTCAGTGTAGTGACCCTTGGC-3') [10]. Amplicons of ITS and $\beta$-tubulin were sequenced and then identified using the BLASTN. Antifungal susceptibilities were determined using the broth dilution method, as recommended by the Clinical and Laboratory Standards Institute (CLSI) M38-A2 (2008) [11]. Antifungal susceptibility testing of the $A$. fumigatus revealed high minimal inhibitory concentrations (MICs) to both itraconazole (ITZ) and PCZ (MICs were $>16$ and $4 \mu \mathrm{g} / \mathrm{mL}$ ), while VCZ MIC revealed a susceptible upper limit $(1 \mu \mathrm{g} / \mathrm{mL})$. Chest $\mathrm{X}$-ray showed steady improvement, and intravenous VCZ was changed to VCZ tablets (200 mg q12 h) and maintained. Therapeutic drug monitoring of $\mathrm{VCZ}$ was performed every week, and it was maintained within therapeutic range between 1.2 and $4.2 \mu \mathrm{g} / \mathrm{mL}$. Thereafter, the patient's absolute neutrophil counts were declined, and he experienced repeated neutropenic fever. On the 69th day of hospitalization (HD 69), the patient died due to $K P C$-producing carbapenemresistant Klebsiella pneumoniae bacteremia which was thought to be associated with concurrent gut GVHD. Previous lesions of IPA were decreased in size (from 5.4 to $1.3 \mathrm{~cm}$ ) at that time. Subsequently, the $A$. fumigatus isolate was analyzed for any known azoleresistant mutations in CYP51A gene. The amplification and sequencing of CYP51A promoter were performed using AFTR-F (5'-TAATCGCAGCACCACTTCAG-3') and AFTR-R (5'-GCCTAGGACAAGGACGAATG-3') [12]. Their CYP51A and promoter sequences were compared to that of an azole-susceptible $A$. fumigatus strain (GenBank accession no. AF338659). Including TR34/L98H, multiple mutations including S297T in the CYP51A gene were identified as shown in Table 1.

\section{Discussion}

This case is that of culture-positive IPA with probable category in an HSCT recipient, which developed 
Table 1 In vitro antifungal susceptibility profile and CYP51A amino acid substitutions in A. fumigatus isolates in this case

\begin{tabular}{|c|c|c|c|c|c|c|c|c|c|}
\hline \multirow{2}{*}{$\begin{array}{l}\text { Source of } \\
\text { isolate }\end{array}$} & \multirow{2}{*}{$\begin{array}{l}\text { PCZ exposure } \\
\text { (days) }\end{array}$} & \multicolumn{4}{|c|}{ Antifungal MIC $(\mu \mathrm{g} / \mathrm{mL})$} & \multirow{2}{*}{$\begin{array}{l}\text { MEC } \\
\text { CAS }\end{array}$} & \multicolumn{3}{|c|}{ CYP51A amino acid substitutions } \\
\hline & & ITZ & VCZ & PCZ & AMB & & TR34 & Codon98 & Others \\
\hline $\begin{array}{l}\text { Bronchial } \\
\text { washing fluid }\end{array}$ & 87 & $>16$ & 1 & 4 & 0.5 & 0.06 & + & L98H & $\begin{array}{l}\text { Y46F, V172 M, T248 N, E255D, } \\
\text { S297T, K427E, F495L }\end{array}$ \\
\hline
\end{tabular}

PCZ posaconazole, MIC minimum inhibitory concentration, MEC minimum effective concentration, ITZ itraconazole, VCZ voriconazole, $A M B$ amphotericin $\mathrm{B}, C A S$ caspofungin

during PCZ prophylaxis due to GVHD. Isolated $A$. fumigatus showed a high MIC for ITZ and PCZ, and CYP51A gene analysis identified a TR34/L98H mutation. In Korea, antifungal susceptibility epidemiology studies in Aspergillus spp. [13] have been reported, but no known gene mutation has been found. This is the first case of IPA caused by TR34/L98H azole-resistant A. fumigatus in Korea.

Based on a systematic study of azole resistance rates and mechanisms conducted by the Nijmegen group in the Netherlands, TR34/L98H is the most common mutation found in clinical and environmental azole-resistant A. fumigatus isolates across Europe [7, 14]. Environmental isolates (soil samples from gardens, plant seeds, hospital surroundings, aerial samples of hospitals) harboring TR34/L98H exhibit resistance to azole fungicides, which are chemically similar to medical triazoles, showing cross-resistance [15]. Therefore, azole fungicides used in the environment have been suggested to induce azole resistance of Aspergillus spp. clinical isolates [4, 15]. Furthermore, close genotypic accordance between A. fumigatus environmental and clinical isolates has been demonstrated, and many patients harbor a single dominant resistance mechanism, even though they are azolenaive or epidemiologically unrelated $[4,6,15]$. Therefore, it has been suggested that the main route of resistance is an acquisition from environmental source [4]. Strains with the TR34/L98H mutation of CYP51A gene also frequently harbor non-synonymous mutations in the CYP51A gene. Some of them are presented alone or in combination, which are known to be associated with higher MICs than the wild type [5, 7].

In this case, isolated A. fumigatus showed relatively higher PCZ MIC ( $4 \mu \mathrm{g} / \mathrm{mL})$ and lower VCZ MIC $(1 \mu \mathrm{g} / \mathrm{mL})$ compared to previously reported MICs of TR34/L98H strains [16]. Also, multiple amino acid substitutions including S297T (Table 1) were found in our isolate. Although most of these substitutions are not clearly characterized with phenotypic relationships, a recent report has suggested that S297T substitution in TR34/L98H strains might represent a compensatory mutation showing this low VCZ MIC [17]. Although there have been no data on the azole resistance of A. fumigatus environmental and clinical isolates in Korea, our hypothesis on the resistance mechanism of the ITZ- and PCZ-resistant strain in this case is a combination of environmental acquisition and the acquired resistance from the patient, probably due to long-term PCZ exposure.

In a national survey conducted on aspergillosis in the Netherlands, where epidemiology data are relatively well established, azole resistance rates have been reported between 5 and $10 \%$, and up to $30 \%$ in high-risk wards [18]. VCZ is still the first drug of choice of IA, even in the clinical setting of high resistance. There are no clear guidelines for azoleresistant and/or breakthrough IA [18]. There are only expert opinions that change the antifungal class to another or the combination of VCZ plus echinocandin when the resistance threshold exceeds $10 \%$ or in the case of azole breakthrough IA [1, 18, 19].

Interestingly, this is an IPA case accompanying influenza A pneumonia, either. Influenza has been established a risk factor for IA since 2009 pandemic influenza $\mathrm{A} / \mathrm{H} 1 \mathrm{~N} 1$ based on many case reports [20-24]. Influenza-associated IA has also been reported in Korea, even in immunocompetent patients $[22,23]$. The interval between the diagnosis of influenza and that of IA varies, and some cases have been diagnosed almost simultaneously as in this case. Although a clear mechanism between influenza and IA has not yet been elucidated, cell-mediated defects, disruption of normal ciliary clearance, or virusinduced host adaptive immunity deficiency model has been proposed [22-25]. Influenza-associated IA showed high mortality rate of $47-61 \%$ in a recent study [24]. However, the impact of concomitant 
influenza pneumonia with IPA on mortality is not clear in this case, since the direct cause of death appears to be the K. pneumoniae bacteremia lasted until the day before death.

Mortality rates of patients with culture-positive azole-resistant IA have been reported as $50-100 \%$, higher than those of azole-susceptible IA $[4,5,26]$. In addition, there are only limited treatment options in cases where azole breakthrough IA is increasing as in the present case. Currently, A. fumigatus susceptibility tests including MICs and CYP51A mutations are not routinely available in most clinical settings. Therefore, azole resistance prevalence surveillance studies are needed for establishing the best choice of treatment. In Korea, the first case has been reported, and further epidemiological surveillance studies are warranted.

Acknowledgements This study was supported by a Grant from the Korean Health Technology R\&D Project, Ministry of Health and Welfare, Republic of Korea (Grant No. HI15C2259).

\section{Compliance with Ethical Standards}

Conflict of interest The authors declare that they have no conflict of interest.

Ethical Standards This study was approved by the Institutional Review Board of Seoul St. Mary's Hospital at the Catholic University of Korea with a waiver of informed consent (IRB No. KC16SISI0307) due to anonymous and retrospective design of the study.

Open Access This article is distributed under the terms of the Creative Commons Attribution 4.0 International License (http:// creativecommons.org/licenses/by/4.0/), which permits unrestricted use, distribution, and reproduction in any medium, provided you give appropriate credit to the original author(s) and the source, provide a link to the Creative Commons license, and indicate if changes were made.

\section{References}

1. Verweij PE, Ananda-Rajah M, Andes D, Arendrup MC, Brüggemann RJ, Chowdhary A, et al. International expert opinion on the management of infection caused by azoleresistant Aspergillus fumigatus. Drug Resist Updat. 2015;21-22:30-40.

2. Kim SB, Cho SY, Lee DG, Choi JK, Lee HJ, Kim SH, et al. Breakthrough invasive fungal diseases during voriconazole treatment for aspergillosis: A 5-year retrospective cohort study. Med Mycol. 2017;55:237-45.

3. Verweij PE, Chowdhary A, Merlchers WJ, Meis JF. Azole Resistance in Aspergillus fumigatus: can we retain the clinical use of mold-active antifungal azoles? Clin Infect Dis. 2016;62(3):362-8.

4. Snelders E, van der Lee HA, Kuijpers J, Rijs AJ, Varga J, Samson RA, et al. Emergence of azole resistance in Aspergillus fumigatus and spread of a single resistance mechanism. PLoS Med. 2008;5:e219.

5. Chowdhary A, Sharma C, Meis JF. Azole-resistant aspergillosis: epidemiology, molecular mechanisms, and treatment. J Infect Dis. 2017;216:S436-44.

6. Chowdhary A, Kathuria S, Xu J, Meis JF. Emergence of azole-resistant Aspergillus fumigatus strains due to agricultural azole use creates an increasing threat to human health. PLoS Pathog. 2013;9:e1003633.

7. Hagiwara D, Watanabe A, Kamei K, Goldman GH. Epidemiological and genomic landscape of azole resistance mechanisms in aspergillus fungi. Front Microbiol. 2016;7:1382.

8. De Pauw B, Walsh TJ, Donnelly JP, Stevens DA, Edwards JE, Calandra T, et al. Revised definitions of invasive fungal disease from the European Organization for Research and Treatment of Cancer/Invasive Fungal Infections Cooperative Group and the National Institute of Allergy and Infectious Diseases Mycoses Study Group (EORTC/MSG) Consensus Group. Clin Infect Dis. 2008;46:1813-21.

9. Toju H, Tanabe AS, Yamamoto S, Sato H. High-coverage ITS primers for the DNA-based identification of ascomycetes and basidiomycetes in environmental samples. PLoS ONE. 2012;7:e40863.

10. Glass NL, Donaldson GC. Development of primer sets designed for use with the PCR to amplify conserved genes from filamentous ascomycetes. Appl Environ Microbiol. 1995;61:1323-30.

11. Clinical and Laboratory Standards Institute. Reference Method for Broth Dilution Antifungal Susceptibility Testing of Filamentous Fungi; Approved Standard-Second Edition. CLSI document M38-A2. Wayne, PA: Clinical and Laboratory Standards Institute; 2008.

12. Morio F, Aubin GG, Danner-Boucher I, Haloun A, Sacchetto E, Garcia-Hermoso D, et al. High prevalence of triazole resistance in Aspergillus fumigatus, especially mediated by TR/L98H, in a French cohort of patients with cystic fibrosis. J Antimicrob Chemother. 2012;67:1870-3.

13. Heo MS, Shin JH, Choi MJ, Park YJ, Lee HS, Koo SH, et al. Molecular identification and amphotericin B susceptibility testing of clinical isolates of Aspergillus from 11 hospitals in Korea. Ann Lab Med. 2015;35:602-10.

14. Koehler P, Hamprecht A, Bader O, Bekeredjian-Ding I, Buchheidt D, Doelken G, et al. Epidemiology of invasive aspergillosis and azole resistance in patients with acute leukaemia: the SEPIA Study. Int J Antimicrob Agents. 2017;49:218-23.

15. Snelders E, Huis In 't Veld RA, Rijs AJ, Kema GH, Melchers WJ, Verweij PE. Possible environmental origin of resistance of Aspergillus fumigatus to medical triazoles. Appl Environ Microbiol. 2009;75:4053-7.

16. van Ingen J, van der Lee HA, Rijs TA, Zoll J, Leenstra T, Melchers WJ, et al. Azole, polyene and echinocandin MIC distributions for wild-type, TR34/L98H and TR46/Y121F/ T289A Aspergillus fumigatus isolates in The Netherlands. J Antimicrob Chemother. 2015;70:178-81. 
17. Deng S, Zhang L, Verweij PE, Tsui KM, Hagen F, Houbraken J, et al. Triazole phenotypes and genotypic characterization of clinical Aspergillus fumigatus isolates in China. Emerg Microbes Infect. 2017;6(12):e109.

18. Lestrade PP, Meis JF, Arends JP, van der Beek MT, de Brauwer E, van Dijk K, et al. Diagnosis and management of aspergillosis in The Netherlands: a national survey. Mycoses. 2016;59:101-7.

19. Patterson TF, Thompson GR 3rd, Denning DW, Fishman JA, Hadley S, Herbrecht R, et al. Practice guidelines for the diagnosis and management of aspergillosis: 2016 update by the infectious diseases society of America. Clin Infect Dis. 2016;63:e1-e60.

20. Garcia-Vidal C, Barba P, Arnan M, Moreno A, Ruiz-Camps I, Gudiol C, et al. Invasive aspergillosis complicating pandemic influenza A (H1N1) infection in severely immunocompromised patients. Clin Infect Dis. 2011;53(6):e16-9.

21. Lat A, Bhadelia N, Miko B, Furuya EY, Thompson GR 3rd. Invasive aspergillosis after pandemic (H1N1) 2009. Emerg Infect Dis. 2010;16(6):971-3.
22. Kim MJ, Kim MK, Kang CK, Jun KI, Bang JH, Park SW, et al. A case of acute cerebral aspergillosis complicating influenza A/H1N1pdm 2009. Infect Chemother. 2013;45(2):225-9.

23. Kwon OK, Lee MG, Kim HS, Park MS, Kwak KM, Park $\mathrm{SY}$. Invasive pulmonary aspergillosis after influenza a infection in an immunocompetent patient. Tuberc Respir Dis Seoul. 2013;75(6):260-3.

24. van de Veerdonk FL, Kolwijck E, Lestrade PP, Hodiamont CJ, Rijnders BJ, van Paassen $\mathbf{J}$ et al. Influenza-associated aspergillosis in critically ill patients. Am J Respir Crit Care Med. 2017 Apr 7. https://doi.org/10.1164/rccm.2016122540le (Epub ahead of print).

25. Bermejo-Martin JF, Martin-Loeches I, Rello J, Antón A, Almansa R, Xu L, et al. Host adaptive immunity deficiency in severe pandemic influenza. Crit Care. 2010;14(5):R167.

26. van der Linden JW, Snelders E, Kampinga GA, Rijnders BJ, Mattsson E, Debets-Ossenkopp YJ, et al. Clinical implications of azole resistance in Aspergillus fumigatus, The Netherlands, 2007-2009. Emerg Infect Dis. 2011;17:1846-54. 